\title{
Téoros
}

Revue de recherche en tourisme

\section{Pourquoi avons-nous besoin de connaître l'expérience de visite des visiteurs des parcs nationaux ?}

\section{Julianna Priskin et Daniel Gosselin}

Volume 25, numéro 3, automne 2006

URI : https://id.erudit.org/iderudit/1071022ar

DOI : https://doi.org/10.7202/1071022ar

Aller au sommaire du numéro

Éditeur(s)

Université du Québec à Montréal

ISSN

0712-8657 (imprimé)

1923-2705 (numérique)

Découvrir la revue

Citer cette note

Priskin, J. \& Gosselin, D. (2006). Pourquoi avons-nous besoin de connaître l'expérience de visite des visiteurs des parcs nationaux ? Téoros, 25(3), 69-72. https://doi.org/10.7202/1071022ar d'utilisation que vous pouvez consulter en ligne. 


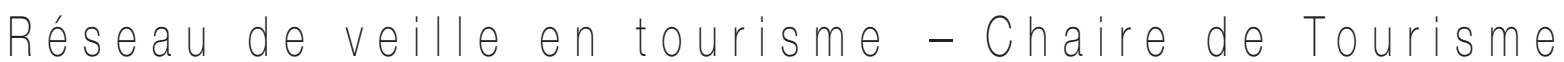 \\ Pourquoi avons-nous besoin de connaître l'expérience de visite des visiteurs des parcs nationaux?}

\section{Julianna Priskin et Daniel Gosselin}

Depuis quelques années, le mot « expérience " a été utilisé à toutes les sauces, créant une certaine confusion quant à sa signification réelle et à son importance pour les besoins de la gestion des aires protégées. En dépit de son aspect nébuleux, la compréhension de ce qu'est l'expérience de visite de ceux qui fréquentent les parcs nationaux est très utile pour la gestion des aires protégées, comme on peut le voir depuis plus de 30 ans. Cet article, dont le but est d'évaluer la relation entre les expériences de visite et la gestion des aires protégées, donnera un aperçu de ce que l'on entend par expérience de visite et présentera certaines mesures pratiques pour la gestion des parcs nationaux.

Dans une optique plus large, il importe de reconnaître que toutes les aires protégées s'inscrivent dans une dynamique sociopolitique qui requiert une planification et une gestion prudente et attentive (Eagles et McCool, 2004). II existe de nombreux types d'aires protégées à travers le monde, mais I'Union mondiale pour la nature classe la plupart des parcs nationaux dans la catégorie II. Cela signifie que l'objectif d'un parc national est la conservation des écosystèmes tout en permettant la pratique d'activités de loisirs. Par conséquent, la gestion des parcs nationaux comporte un double mandat. En pratique, elle exige, d'une part, une conservation des écosystèmes garantissant un haut degré d'intégrité écologique et, d'autre part, un accès du grand public aux parcs nationaux pour des activités de loisirs. Cela signifie que l'on doit être en mesure de satisfaire les besoins fondamentaux des gens, tout en leur fournissant, progressivement, les moyens de vivre une expérience de visite de haute qualité (Roggenbuck, 2000 ; Lime et al., 2004). La gestion des parcs est donc une tâche difficile, car toutes les activités de loisirs peuvent avoir des répercussions négatives sur l'état de l'écosystème. De son côté, l'état d'un écosystème peut influencer l'expérience de visite des visiteurs. Depuis les années 1960, la recherche portant sur les expériences de divertissement dans les aires protégées continue d'intéresser de nombreuses disciplines comme la science des loisirs, la psychologie et, de plus en plus, le marketing (Watson et Williams, 1995 ; Glaspell et Puttkammer, 2001).

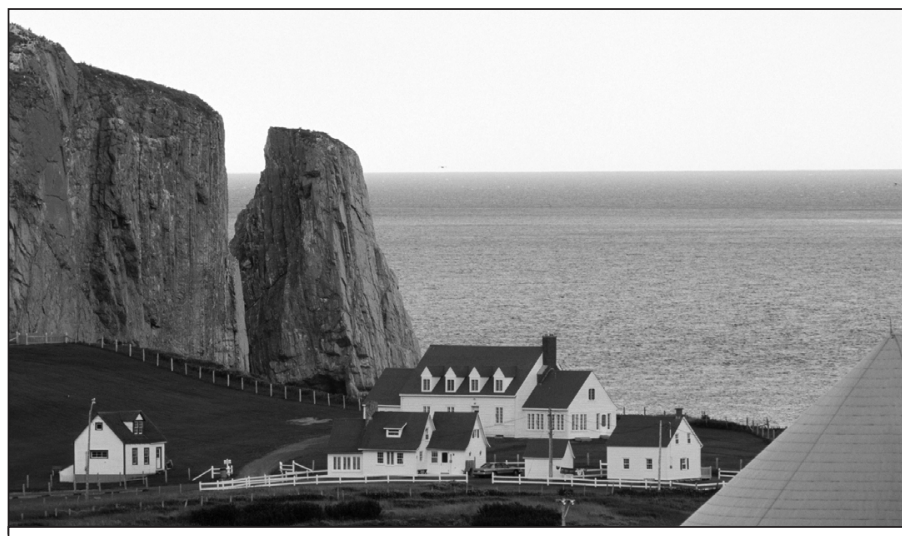

Rocher Percé au Parc national de l'Île-Bonaventure-et-du-Rocher-Percé dans la région touristique de la Gaspésie (Québec).

Photo: Sylvain Majeau/Tourisme Québec

\section{En quoi consiste l'expérience de visite d'un visiteur ?}

Au départ, il faut dire qu'une expérience humaine, quelle qu'elle soit, comporte une multiplicité de facteurs. Quel que soit le point de vue que l'on adopte, l'expérience humaine est propre à chaque individu et engage tous les sens (Ryan, 2002 : 27). Cela confère au travail sur l'expérience humaine une dimension subjective d'une grande complexité, car toutes les expériences individuelles des mêmes endroits et des mêmes événements se font suivant une construction de la réalité qui est propre à chacun et à chacune. Cette situation, à son tour, dépend des nombreux facteurs qui influencent les processus cognitifs (Botterill et Crompton, 1996). Ce qu'une personne qui se rend dans un parc national considère comme une expérience divertissante pourra très bien être vu comme ennuyeux ou stimulant, voire effrayant, par une autre. L'expérience de visite de chacune des personnes qui fréquentent un parc national varie en fonction du moment et du lieu où elle se trouve. Dès lors, il est possible de définir l'expérience de visite dans un parc national comme «l'interaction complexe entre les personnes et leurs états d'âme, l'activité dans laquelle elles sont engagées et le milieu naturel et social où elles se trouvent » (Borrie et Roggenbuck, 1998: 115).

Cependant, il convient de souligner qu'une activité quelconque ne constitue pas en elle-même une "expérience de visite ». Les gens disent volontiers des choses comme «mon expérience en canoë fut extraordinaire ". À vrai dire, le canotage n'est qu'une activité ou une manière de s'engager en vue de vivre une expérience personnelle « extraordinaire ". II est donc une interaction entre un parc et un visiteur, le 
niveau de participation physique, cognitive et émotionnelle dans cette activité déterminant l'intensité de l'expérience qui en résultera pour la personne concernée. Cela peut varier énormément d'un individu à l'autre : les activités contribuent diversement à l'expérience de visite de chacun des visiteurs d'un parc national. Ainsi, l'expérience d'une personne faisant de la randonnée sera différente de celle vécue par un cycliste de montagne, même si ces activités se produisent sur le même sentier (et même si elles sont pratiquées par le même visiteur, placé dans des conditions physiques, sociales et une situation de gestion identiques) (Roggenbuck, 2000).

Ce qui distingue une expérience en général de l'expérience de visite est le fait que cette dernière suppose un déplacement spécifique à un moment précis, à des fins (en règle générale) de loisirs ou de tourisme. L'expérience de visite peut donc se comprendre comme un processus comportant de nombreuses étapes, par exemple la perspective de la visite, les déplacements pour se rendre sur les lieux et pour en revenir, la visite du parc et le souvenir qu'il en garde (Clawson et Knetsch, 1966 ; Hammitt, 1978; Lee et al. 1994).

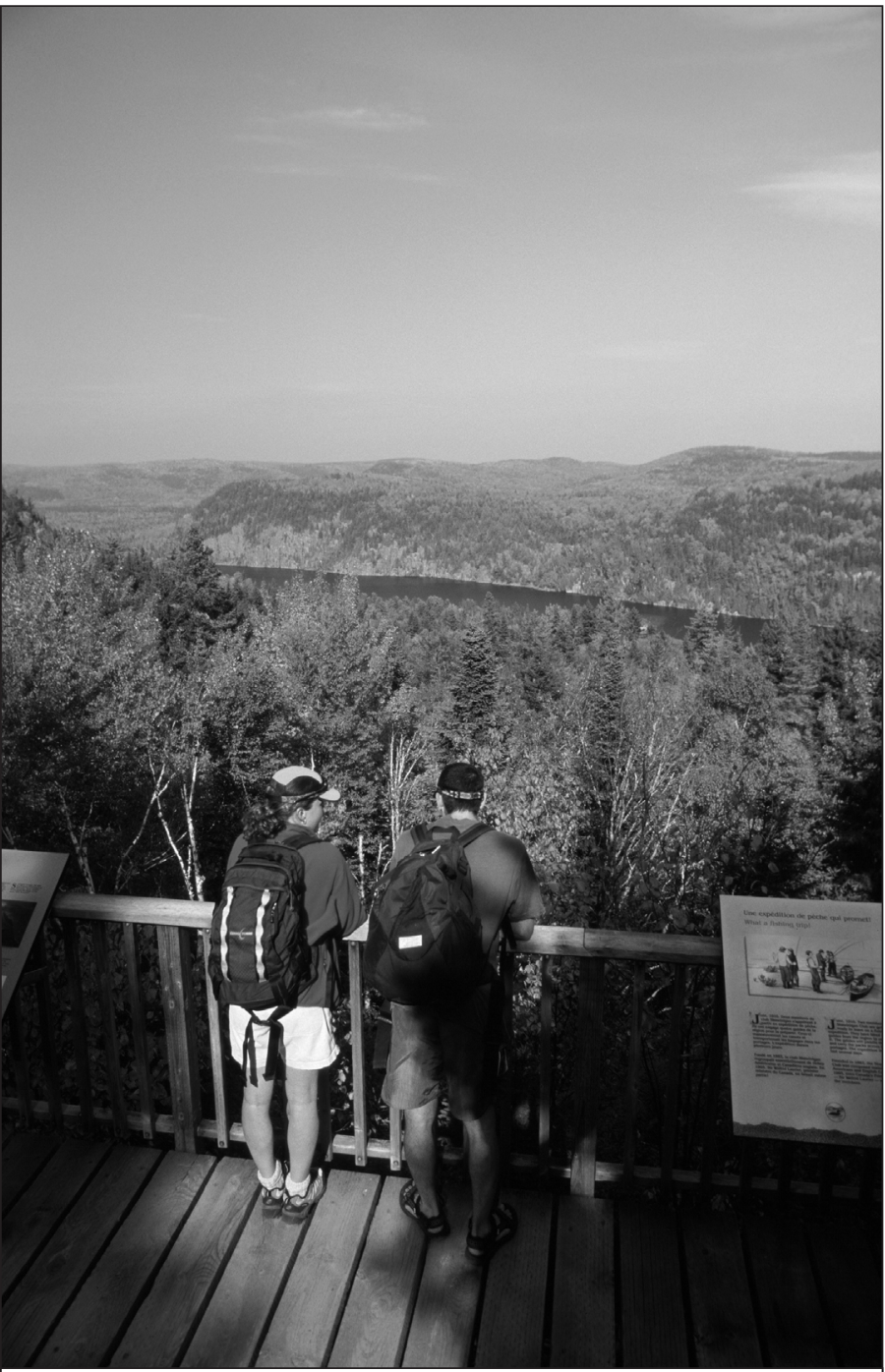

Randonnée pédestre et écotourisme dans le Parc national du Canada de la Mauricie (Québec).

Photo: Paul Villecourt/Tourisme Québec
Les connaissances concernant les expériences de divertissement dans les aires protégées ont sensiblement évolué au cours des décennies, même si la description précise des phénomènes en cause pose encore plusieurs défis (Roggenbuck, 2000). Les expériences de divertissement ont été étudiées suivant plusieurs approches qui, toutes, fournissent des résultats différents. Par exemple, les études qui s'appuient sur la motivation et la psychologie ont montré que c'est l'expérience vécue qui explique pourquoi les gens recherchent certains types d'activités de loisirs (Manfredo et al., 1996). Cela donne une idée de ce que les gens attendent de leurs expériences de séjour, ainsi que des avantages qu'ils en retirent (Patterson, et al., 1998 ; Lee et Driver, 1996 ; Driver et Burns, 1999).

Même si les gens se livrent à des activités différentes dans le même parc, ils peuvent retirer de leur expérience des bienfaits tout à fait semblables, tels que l'apprentissage, la relaxation, une prise de conscience accrue, etc. Les expériences vécues par les visiteurs peuvent avoir de nombreux avantages individuels aussi bien que collectifs. Ainsi, une activité d'apprentissage effectuée dans le cadre d'une visite d'un parc national peut stimuler la prise de conscience par un individu de la valeur écologique des aires de conservation. D'autres effets peuvent s'ensuivre, comme une modification du comportement dans la vie quotidienne. Une personne pourra se transformer en consommateur responsable et choisir de s'engager activement dans des activités de conservation.

Jusqu'à présent, l'approche privilégiée adoptée pour l'étude des expériences de visite des visiteurs s'est appuyée sur des mesures de la qualité et de la satisfaction (Borrie et Birzell, 2001; Tian-Cole et al., 2002 ; Shelby et al., 1988). Cette approche fournit des réactions utiles sur les préférences et les attentes des visiteurs dans les parcs, ou sur la manière dont leur expérience de visite fut positivement ou négativement influencée par différents facteurs (Dawson et al., 1998 ; Cole 2001b ; Stewart et Cole, 2001).

\section{Mesures pour la gestion des parcs}

Toutes les expériences de visite dans un parc national sont influencées par un nombre indéterminé de facteurs liés aux visiteurs et aux endroits visités. Toutefois, certains facteurs jouent un rôle plus important que d'autres et peuvent même avoir une influence prédominante. Les facteurs dont on doit tenir compte pour un parc national se rapportent à l'intégrité écologique, aux équipements d'infrastructures, aux services, à la condition sociale des visiteurs et à leur gestion (comme le comportement des visiteurs et leur nombre). Plusieurs facteurs sont donc à envisager, le plus important d'entre eux étant souvent les conditions météorologiques.

Les caractéristiques personnelles des visiteurs influencent aussi leurs expériences vécues (Watson et al., 1991). La manière dont chacun décide de faire l'expérience de visite d'un parc national est hautement variable. Les gestionnaires doivent en conséquence connaître le cadre de référence à partir duquel un visiteur évalue son expérience, dans le but d'offrir un large éventail de possibilités d'expériences de visite à partir des types de services et 
d'installations fournis (Ibid.). En réalité, les gestionnaires de parcs ne contrôlent pas toutes les expériences des gens ; cependant, ils peuvent espérer en améliorer certaines par leur style de gestion. Les gestionnaires peuvent offrir aux visiteurs un large éventail d'expériences de visite de grande qualité, comprenant des découvertes, des expériences excitantes, amusantes, enthousiasmantes, intéressantes et une infinité d'autres possibilités. Toutefois, pour que ces expériences puissent être vécues de manière optimale, la compréhension de certaines " expériences-clés à l'intérieur des parcs nationaux » et la connaissance des facteurs qui les influencent sont nécessaires.

La reconnaissance des principales caractéristiques des visiteurs, associée aux expériences-clés, peut également permettre d'apporter aux sous-groupes de visiteurs un soutien qui dépasse les simples moyens traditionnels déployés à partir des caractéristiques démographiques et des activités. Cette démarche peut également comprendre d'autres mesures dans le cadre de la commercialisation de certaines expériences de visite auprès de certains segments de marché.

Étant donné que l'éducation est une préoccupation centrale de l'ensemble des gestionnaires de parcs, il importe de comprendre la dimension d'apprentissage de l'expérience de visite. Par exemple, quels sont les éléments les meilleurs du parc pour favoriser une expérience d'apprentissage? Quel est le pourcentage des clients du parc qui réussissent à atteindre tel ou tel degré d'une expérience d'apprentissage? De quelle manière l'expérience d'apprentissage correspond-elle aux caractéristiques des visiteurs? Comment les gestionnaires peuvent-ils améliorer l'expérience d'un visiteur pour qu'elle corresponde mieux aux valeurs d'une agence de gestion des aires protégées ? Où doivent se rendre les visiteurs qui désirent profiter d'une expérience guidée d'apprentissage dans un parc national?

II est clair que ce ne sont pas toutes les dimensions de l'expérience humaine qui sont du ressort des gestionnaires de parcs nationaux. Les études actuelles à propos des expériences de visite dans les aires protégées s'efforcent encore de découvrir ce que les visiteurs attendent de leur visite et ce qu'elle leur permet de vivre. Les gestionnaires ont également besoin de comprendre quelles sont les expériences les plus importantes, étant admis que les ressources dont ils disposent sont limitées et qu'elles doivent leur permettre de faire face, d'une manière réaliste, à toutes les dimensions de l'expérience de visite. Souvent, il est nécessaire de faire des choix; dans ces conditions, les ressources de gestion doivent être classées par ordre de priorité. Si les gestionnaires de parcs comprennent effectivement quelles caractéristiques d'un parc national peuvent améliorer ou altérer certaines dimensions de l'expérience de séjour, ils peuvent ajuster en conséquence les services offerts et ainsi répondre plus efficacement aux besoins et aux attentes des clients, tout en s'acquittant de leur mandat de protéger l'intégrité écologique d'un parc.

Soulignons un autre point très important : étant donné que les gestionnaires de parcs sont tenus de protéger l'intégrité des écosystèmes, il est indispensable de trouver un équilibre concret entre les expériences désirables du point de vue des gestionnaires et celles qui le sont du point de vue des visiteurs. Les gestionnaires doivent donc comprendre les besoins et les attentes des visiteurs, afin de pouvoir offrir un large éventail d'expériences de visite satisfaisantes. Dans une société qui évolue rapidement, les besoins et les attentes des visiteurs changent constamment ; il incombe aux gestionnaires de fournir des solutions appropriées.

En réalité, l'intégration des données sur les expériences de visite dans la planification et la gestion des parcs nationaux n'est pas une idée nouvelle, puisqu'elle date de la fin des années 1970. Une des structures de planification des espaces naturels les plus connues est le Recreation Opportunity Spectrum (ROS), système qui aide à décrire les expériences possibles dans une aire donnée en fonction de conditions biophysiques, sociales et de contextes de gestion variés (Clark et Stankey, 1979). Or, le ROS donne à penser que le désir de vivre des expériences particulières est lié à la préférence accordée à différents lieux de loisirs. S'appuyant sur des principes semblables, le US National Park Service a élaboré le Visitor Experience Resource Planning (VERP) au cours des années 1990 (Hof et Lime, 1998).

Le cadre de planification du VERP s'intéresse à la gestion de l'usage du parc par les visiteurs et aux questions de capacité de support dans différentes zones d'un parc national. Ce système a été mis au point parce que, de nos jours, de plus en plus de gens visitent les parcs nationaux et que ce phénomène a une incidence sur les ressources naturelles et culturelles des zones concernées, ainsi que sur la qualité de l'expérience vécue par les visiteurs. Cela engendre un conflit entre, d'une part, les efforts des gestionnaires de parcs pour favoriser l'utilisation des parcs et leur accès à un plus grand nombre de gens et, d'autre part, leurs efforts pour s'assurer que les ressources du parc sont protégées, tout en garantissant des possibilités d'expériences de grande qualité aux visiteurs (Hof et Lime, 1998).

La compréhension des expériences de visite dans chacune des zones d'un parc procure aux gestionnaires de l'information qui peut être importante lorsqu'il s'agit de surveiller l'état des ressources ou les expériences des visiteurs (Lime et al., 2004). Par ailleurs, cette compréhension est utile lors de la production de cartes d'expériences à vivre, indiquant les types d'expériences associés aux différentes zones du parc. En outre, la surveillance de l'état du parc et la prise de conscience des expériences de visite contribuent toutes deux à fournir aux gestionnaires des réactions concernant les conséquences de leurs plans d'actions spécifiques. Ces réactions les renseignent sur les répercussions de leurs décisions et les aident à mieux gérer les parcs.

\section{Conclusion}

Étant donné la complexité et le grand nombre de facteurs interactifs qui ont une incidence sur toute expérience de visite des visiteurs, cette notion devra éventuellement être simplifiée pour devenir concrètement opérationnelle aux fins de la gestion des aires protégées. II reste difficile d'établir clairement les relations de causalité entre l'expérience de visite du "visiteur type » et les facteurs individuels, bien que les chercheurs, à l'échelle mondiale, fassent des progrès sensibles dans ce domaine. La clé du succès pourrait être une compréhension globale de 
l'expérience du visiteur, ainsi que des facteurs qui l'influencent et sur lesquels le gestionnaire exerce un contrôle ; car ce dernier n'a pas toujours les moyens ni les compétences requis pour aborder en profondeur les aspects psychologiques de telles expériences. Il est donc important qu'il comprenne quels sont les éléments d'un parc qui sont importants par rapport à des expériences spécifiques et comment les différentes conditions sociales et les différentes situations de gestion peuvent affecter ces expériences.

Julianna Priskin est professeure associée au Département d'études urbaines et touristiques de l'Université du Québec à Montréal et chercheure à la Chaire de Tourisme de la même Université.

Daniel Gosselin est agent de recherche socio-économique à Parcs Canada, Centre de services du Québec.

\section{Bibliographie}

Borrie, W.T., et R.M. Birzell (2001), "Approaches to Measuring the Quality of the Wilderness Experience", dans W.A. Freimund et D.N. Cole (dir.), Visitor Use Density Experience: Proceedings, 1er-juin 2000, Missoula (MT), Proc. RMRS-P-20, Ogden (UT), US Department of Agriculture, Forest Service, Rocky Mountain Research Station, p. 29-38.

Borrie, W.T., et J.W. Roggenbuck (1998), "Describing the Wilderness Experience at Juniper Prairie Wilderness Using Experience Sampling Methods", dans D.L. Kulhavy et M.H. Legg (dir.), Wilderness and Natural Areas in Eastern North America: Research, Management and Planning, Nacogdoches (TX), Stephen F. Austin State University, Arthur Temple College of Forestry, Center for Applied Studies, p. 165-172.

Botterill, T.D., et J.L. Crompton (1996), "Two Case Studies Exploring the Nature of Tourist's Experience ", Journal of Leisure Research, vol. 28, nº 1, p. 57-82.

Clark, R.N., et G.H. Stankey (1979), The Recreation Opportunity Spectrum: A Framework for Planning, Management and Research, US Department of Agriculture and Forest Service, GTR-PNW 98, Pacific NW Forest and Range Station, 1979, 32 p.

Clawson, M., et J.L. Knetsch (1966), Economics of Outdoor Recreation, Baltimore (MD), Johns Hopkins Press, 1966, cité dans W.E. Hammitt (1978), "Outdoor Recreation: Is It a Multi-phase Experience? », Journal of Leisure Research, vol. 12, n², p. 107-115.

Cole, D.N. (2001), "Visitor Use Density and Wilderness Experiences: A Historical Review of Research ", dans W.A. Freimund et D.N. Cole (dir.), Visitor Use Density Experience: Proceedings, $1^{\mathrm{er}}-3$ juin 2000, Missoula (MT), Proc. RMRS-P-20, Ogden (UT), US Department of Agriculture, Forest Service, Rocky Mountain Research Station, p. 11-20.

Dawson, C.P., P. Newman, et A. Watson (1998), " Cognitive Dimensions of Recreational User Experiences in Wilderness: An Exploratory Study in Adirondack Wilderness Areas ", dans H.G. Vogelson (dir.), Proceedings of the 1997 Northeastern Recreation Research Symposium, 6-9 avril 1997, New York, Bolton Landing, 1998. Gen. Tech. Rep. NE-241, Radnor, Philadelphia, U.S. Department of Agriculture, Forest Service, Northeastern Forest Experiment Station, $287 \mathrm{p}$.

Driver, B.L., et D.H. Burns (1999), "Concepts and Uses of the Benefits Approach to Leisure ", dans E.L. Jackson et T.L. Burton (dir.) (1999), Leisure Studies, Prospects for the $21^{\text {st }}$ Century, Venture Publishing, p. 349-369.

Eagles, P.F.J., et S.F. McCool (2004), Tourism in National Parks and Protected Areas. Planning and Management, Wallingford, CABI Publishing, $320 \mathrm{p}$.
Glaspell, B., A. Watson, K. Kneeshaw, et D. Pendergrast (2003), « Selecting Indicators and Understanding their Role in Wilderness Experience Stewardship at Gates of the Arctic National Park and Preserve ", George Wright Forum, vol. 20, n 2, p. 59-71.

Hammitt, W.E. (1978), « Outdoor Recreation: Is It a Multi-phase Experience? », Journal of Leisure research, vol. 12, n² 2, p. 107-115.

Hof, M., et D.W. Lime (1998), «Visitor Experience and Resource Protection Framework in the National Park System: Rationale, Current Status, and Future Direction ", dans S.F. McCool et D.N. Cole (dir.), Proceedings Limits of Acceptable Change and Related Planning Processes: Progress and Future Directions, 20-22 mai 1997, Missoula (MT), Report INT-GTR371, Department of Forest Service, Rocky Mountain Research Station, Ogden (UT), p. 29-36.

Lee, Y., J. Dattilo, et D. Howard (1994), "The Complex and Dynamic Nature of the Leisure Experience ", Journal of Leisure Research, vol. 26, $n^{\circ}$ 3, p. 195-211.

Lee, M., et B. Driver (1996), «Benefits-based Management: A New Paradigm for Managing Amenity Resources ", dans W. Burch, J. Aley, B. Conover et D. Field (dir.), Survival of the Organizationally Fit: Ecosystem Management as an Adaptive Strategy for Natural Resource Organizations in the $21^{\text {st }}$ century, New York, Taylor and Francis.

Lime, D.W., D.H. Anderson, et J.L. Thompson (2004), Identifying and Monitoring Indicators of Visitor Experience and Resource Quality. A Handbook for Recreation Resource Managers, University of Minnesota, Department of Forest Resources, St. Paul (MN), 53 p.

Manfredo, M.J., B.L. Driver, et M.A. Tarrant (1996), "Measuring Leisure Motivation: A Meta-analysis of the Recreation Experience Preference Scales ", Journal of Leisure Research, vol. 28, p. 188-213.

Patterson et al., 1998. "An Hermeneutic Approach To Studying the Nature of Wilderness Experiences ", Journal of Leisure Research, vol. 30, n 4 , p. $423-452$.

Roggenbuck, J.W. (2000), "Facilitating High-quality Visitor Experiences at National Wildlife Refuges ", dans D.C. Fulton, K.C. Nelson, H. Anderson et D.W. Lime (dir.), Human Dimensions of Natural Resource Management: Emerging Issues and Practical Applications, Cooperative Park Studies Program, St. Paul, University of Minnesota.

Ryan, C., (2002), The Tourist Experience, Londres, Continuum, 233 p.

Shelby, B., N.S. Bregenzer, et R. Johnson (1988), "Displacement and Product Shift: Empirical Evidence from Oregon Rivers ", Journal of Leisure Research, vol. 20, n 4, p. 274-288.

Stewart, W.P., et D.N. Cole (2001). « Number of Encounters and Experience Quality in Grand Canyon Backcountry: Consistently Negative and Weak Relationships ", Journal of Leisure Research, vol. 33, n 1, p. 106-120.

Tian-Cole, S., J.L. Crompton, et V.L. Willson (2002), Journal of Leisure Research, vol. 34, n¹, p. 1-24.

Watson, A.E., J.W. Roggenbuck, et D.R. Williams (1991), «The Influence of Past Experience on Wilderness Choice ", Journal of Leisure Research, vol. 23, n०1, p. 21-36.

Watson, A.E., et D.R. Williams (1995), "Priorities for Human Experience Research in Wilderness", Trends/Wilderness Research, vol. 32, nº 1 , p. $12-18$. 